FedUni ResearchOnline http://researchonline.federation.edu.au

This is the peer-reviewed version of the following article:

Kaisar, S., Kamruzzaman, J., Karmakar, G., \& Gondal, I. (2016). Carry me if you can: A utility based forwarding scheme for content sharing in tourist destinations. 22nd Asia-Pacific Conference on Communications, APCC 2016; Yogyakarta, Indonesia; 25th-27th August 2016, Institute of Electrical and Electronics Engineers Inc. 261-267.

Which has been published in final form at:

https://doi.org/10.1109/APCC.2016.7581432

(C) Copyright 2017 IEEE - All rights reserved. Personal use of this material is permitted. Permission from IEEE must be obtained for all other uses, in any current or future media, including reprinting/republishing this material for advertising or promotional purposes, creating new collective works, for resale or redistribution to servers or lists, or reuse of any copyrighted component of this work in other works. 


\section{Carry Me if You Can: A Utility Based Forwarding Scheme for Content Sharing in Tourist Destinations}

\author{
Shahriar Kaisar* \\ *Faculty of Information Technology \\ Monash University, Australia \\ Email:shahriar.kaisar@monash.edu
}

\author{
Joarder Kamruzzaman $^{\dagger *}$, Gour Karmakar ${ }^{\dagger}$ and Iqbal Gondal ${ }^{\dagger *}$ \\ ${ }^{\dagger}$ School of Engineering and Information Technology \\ Federation University Australia \\ Email:\{joarder.kamruzzaman, gour.karmakar, Iqbal.gondal\}@ federation.edu.au
}

\begin{abstract}
Message forwarding is an integral part of the decentralized content sharing process as the content delivery success highly depends on it. Existing literature employs spatiotemporal regularity of human movement pattern and pre-existing social relationship to take message forwarding decisions. However, such approaches are ineffectual in environments where those information are unavailable such as a tourist spot or camping site. In this study, we explore the message forwarding techniques in such environments considering the information that are readily available and can be gathered on the fly. We propose a utility based forwarding scheme to select the appropriate forwarder node based on co-location stay time, connectivity and available resources. A higher co-location stay time reflects that the forwarder and the destination node is likely to have more opportunistic contacts, while the connectivity and available resource ensure that the selected forwarder has sufficient neighbours and resources to carry the message forward. Simulation results suggest that the proposed approach attains high hit and success rate and low latency for successful content delivery, which is comparable to those proposed for work-place type scenarios with regular movement pattern and pre-existing relationships.
\end{abstract}

\section{INTRODUCTION}

The popularity of smart mobile devices is growing at a rapid speed. A recent report from Cisco suggests that within 2020, 67 percent of the mobile devices will be smart devices, which is an increment of 31 percent from the year 2015 [1]. Smart mobile devices enable users to generate and share various types of contents such as music, videos, photos and news items. Content sharing (CS) using smart mobile devices mostly utilize a centralized or hybrid platforms (e.g., platforms that use a central server to store and deliver contents) which provide a reliable and timely service, but introduce problems such as (i) constant requirement of Internet connection whose availability cannot be always guaranteed at all places, (ii) increment of Internet traffic which is already approaching a bottleneck [2], and (iii) high cost of Internet connection if accessed through cellular network. As an alternative, decentralized content sharing (DCS) approach is proposed in the literature, where nodes (mobile devices) opportunistically connects with each other and deliver contents employing a peer-to-peer architecture. DCS approach does not require an Internet connection, does not add to Internet traffic or incur cost.

In DCS approach, nodes are usually grouped into communities (i.e., groups) based on some pre-defined criteria (e.g., mutual interest, proximity). One of the community members acts as an administrator, who stores and maintains a content list summary of all the community members, and provides directory services. Whenever any node requests for a content, the request is forwarded to the content holder by its neighbours and the administrator. Later, the content holder tries to deliver the content to the requester through neighbour nodes. Content delivery success depends on locating the content holder(s) and finding a routing path between the holder and the requester. In content sharing, the network is made of mobile devices carried by human beings who create a highly dynamic network topology. The traditional message forwarding approaches developed for wireless networks which require a connected network are not applicable here, as there might not be any end-to-end path from the content holder to the requester. To address this, store-carry-and-forward techniques proposed for delay tolerant networks (DTN) are employed where a node should carry a message until it finds a suitable forwarder node or the lifetime of the message expires if the destination is unreachable [3]. Although, DCS approach is slightly different from DTN approaches, as DCS requires locating the content before delivering it to the requesters, the message forwarding techniques developed for DTN can be modified to accommodate content delivery.

One of the basic message forwarding schemes used for DTN is called epidemic routing [4], where a node carrying a message forward it to every other nodes it meets who does not have a copy. Although epidemic routing yields most successful deliveries, it requires high amount of resources and creates unnecessary replication which, in turn, increases network congestion. To alleviate this situation several message forwarding techniques are proposed in the literature that can be broadly categorized into mobility pattern based [3], [5] and social relationship based [6], [7] message forwarding techniques. In mobility pattern based forwarding techniques, it is assumed that nodes follow a predictable regular movement pattern and usually visit a particular set of locations in a predictable sequence [3], [5]. Such information is exploited to calculate meeting probability with the destination and forwarding decisions. Social relationship based message forwarding techniques consider that people more often meet a particular set of people on a regular basis and shows stronger social ties [6], [7]. Some of the approaches collect this information from existing social networks and others build it over time based on meeting frequency.

Most of the existing approaches either consider a predictable regular movement pattern or pre-existing social relationship for message forwarding decisions. Such approaches 
are suitable for sharing contents in work-place type scenarios, for example, among students in a university campus or colleagues in an office. However, these approaches are not directly applicable in scenarios where people mostly meet strangers and do not exhibit a regular movement pattern such as a tourist destination or camping site. Content sharing in these environments can be beneficial as many tourist spots lack Internet connectivity and the shared content can be used for information sharing and entertainment purposes which will enrich the tourists' experience. A few recent approaches [8], [9] addressed content sharing in such tourist destinations, however the focus of those works were group formation and administrator selection. Binary spray-and-wait [10] protocol was used for message forwarding which spreads a specific number of copies of a message in the network. In this case, a content holder blindly forwards a message to its neighbours without considering their suitability for successful delivery and hence consumes unnecessary resource or not necessarily contributes to successful delivery. Free mobility of nodes in a tourist spot makes message forwarding more complicated and therefore, intelligent selection of forwarding nodes is highly important.

In this paper, we consider a utility based forwarding scheme (UBF) for content delivery, where a content holder considers the utility of its surrounding node to deliver the content and only forward a content when it meets another node with higher utility. The utility measures a node's suitability for carrying data leading to a successful delivery and is calculated using co-location probability, connectivity and available resources. Extensive simulation using network simulator NS3 is performed to validate the outcome of the proposed approach considering a test location in Victoria, Australia. Simulation results suggest that the proposed approach attains higher hit and success rates, and lower latency for delivering content.

\section{RELATED WORK}

Although epidemic routing [4] is simple and maximize successful delivery, it increases overhead by consuming more buffer space, bandwidth and energy. To reduce the overhead of epidemic routing, spray-and-wait [10] mechanism is proposed which spreads a specific number of copies $(X)$ of a message in the network. In binary spray-and-wait method, whenever a node meets another node with $m>1$ copies available for forwarding, it forwards $\left\lfloor\frac{m}{2}\right\rfloor$ copies and keeps $\left\lceil\frac{m}{2}\right\rceil$ copies for itself. If any node has only a single copy available, it holds on to that copy until it meets the destination. However, determining the appropriate value of $X$ in a network with unknown parameters (i.e., total number of nodes) is difficult since the estimation requires periodic contacts which might not be available in short-lived networks such as in a tourist spot. The forwarding mechanism also does not consider the suitability of the forwarder and blindly forwards the message which increases unnecessary overhead. To address this problem utility based message forwarding is proposed in the literature. Utility based message forwarding can be classified into mobility pattern based and social relationship based message forwarding techniques.

Mobility pattern based approaches consider that nodes usually visit a particular set of locations in a predictable sequence on a regular basis which can be utilized for successful message forwarding. PROPHET [11] is one of the prominent works which considers the probability of meeting other nodes based on the frequency of previous encounters. It considers that the higher the meeting frequency, the more likely that these nodes will meet again. However, it does not consider place of meeting. To address this MobySpace [12] and meetings and visits [13] are proposed which use mobility patterns of the users and the places they visit for packet forwarding. Predictand-relay [5] considers that people visit a set of landmarks and the movement between these landmarks is semi-deterministic provided that enough historical information about movement is available. However, none of these works consider the temporal properties of movements. Recently, Talipov et al. [3] have utilized both temporal and spatial regularity of movement pattern while forwarding requests. They proposed adding future time and location of the requester along with the requests which can be used by the recipient to take forwarding decisions. The problem associated with mobility pattern based approaches is that they require a predictable regular movement pattern which most likely is not available in places such as a tourist spot where people goes for random visit and shows spontaneous movements, and most people are strangers to others.

Social relationship based message forwarding is inspired by relationships among participating nodes extracted from online social networks or built over time based on the time they spent together. SimBet [14] uses betweenness centrality and social similarity for taking forwarding decisions. Whenever two nodes meet each other, the node with the lower degree centrality forwards the message to the other node. This approach makes the nodes with higher degree centrality more congested and does not consider relationship with the destination. To address this issue SimbetTS [15] was proposed that uses tie strength with the destination along with betweeneness centrality and similarity for taking forwarding decisions. This approach does not consider community membership for forwarding where nodes might be a part of a specific community and only interested to forwards messages for other members of the same community. Bubble rap [7] is a community based message forwarding approach where messages are forwarded among the community members based on their popularity which is measured in terms of meeting frequency with other community members. In this approach, a node with lower popularity always forwards a message to a node with higher popularity. This approach also makes the most popular node more congested. The social relationship based message forwarding techniques are also not applicable in an environment like tourist spot where people mostly meet strangers and stay for a short duration to allow building meaningful relationship.

Few recent works have considered content sharing in tourist locations [8], [9]. Their focus was group management and administrator selection rather than message forwarding. Binary spray-and-wait [10] was used for message forwarding in those works. However, detection of appropriate number of copies for successful delivery is not addressed. A higher number of copies essentially increases the probability of successful delivery and at the same time consume more resources (e.g., bandwidth, storage, energy). A lower number of copies (e.g. single copy) will result in lower resource consumption and low successful deliveries, if potential forwarders are blindly selected using this approach which lacks consideration of some practical aspects (e.g., connectivity, buffer space, remaining energy). Finally, message forwarding schemes need to ensure that the requester 
and the content holder/forwarder will meet in near future to allow successful delivery which is not addressed at all.

\section{NETWORK MODEL AND PROBLEM STATEMENT}

\section{A. Network Model}

We consider content sharing in a tourist spot where the areas designated for different activities are called POIs which can be detected using any map application and/or online information provided by the visitor information centre. For example, a tourist spot can have areas such as 'Bowling', 'Fishing', 'Camping', 'Accommodation', 'Food and Shopping' and 'Bush walking' which represent activities performed in those areas. Users can enter one of these POIs based on their interest, perform some activities and then move on to the next POI or leave the tourist spot. A node can calculate its own interest score and joins content sharing group based on mutual interest with other nodes. An administrator for each group is selected who is responsible for maintaining and updating group content list (i.e., content list of all group members). When an administrator receives a request for a content, it checks this group content list to determine if any of the group members has a matching content and directs the content request towards that member. Details of interest score calculation for each activity, group formation and administrator selection can be found in our previous work presented in [9]. Whenever a user is interested to obtain a content, it generates a request which is forwarded to the content holder with the administrator's help. A content holder can match a request with its stored content using the methods discussed in [6]. We assume that a requester specifies the lifetime of the request (i.e., after this time it is not interested to obtain the content) and the next activity it is going to perform while sending the request. If the requester does not mention its next activity then we can use historical information or interest score for each activity to predict the next activity.

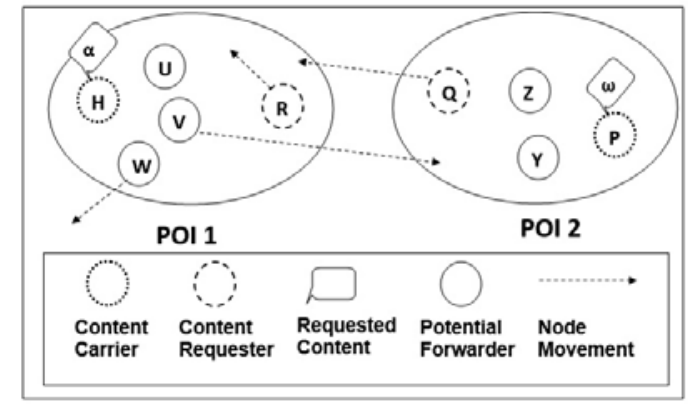

Fig. 1: Message forwarding in content sharing

\section{B. Problem statement}

Figure 1 shows a schematic representation of the message forwarding problem. It shows that, node $H$ is a content carrier node(i.e., content holder or a forwarder) with content $\alpha$ and needs to deliver the content to requester node $R$ which is not within its communication range. Now with single copy message forwarding technique node $H$ needs to decide whether to keep the message (i.e., requested content $\alpha$ ) or to forward it to one of the neighbours within its communication range (i.e.,
$U, V$ or $W$ ). If node $H$ randomly selects one of the nodes (e.g., $V$ or $W$ ) then that node might leave the current POI before delivering the content. Similarly, carrier node $P$ needs to deliver content $\omega$ to requester $Q$; however, in this case the requester itself might move into other POI before obtaining the content. In general, the message forwarding problem can be considered as finding the best node to carry the message for successful delivery whenever a new message is received or a new node is encountered. Note that existing approaches in the literature based on predictable mobility pattern or social relationship are not applicable here as discussed before.

\section{UTILITY BASED FORWARDING SCHEME FOR CS}

When a carrier node (e.g., $H$ and $P$ in Fig. 1) has a content to deliver, it can either carry the content by itself for future delivery or forward the content to one of its neighbours. Successful content delivery relies on the probability of nodes (i.e., both carrier and requester) staying in the same POI and the carrier node having a higher connectivity and enough available resources. Staying in the same POI as the destination node for longer time enhances the carrier's chance of having more opportunistic contact. Similarly, a carrier node with more neighbours has a better probability of delivering the content rather than a stand-alone node. It also needs to have sufficient resources (e.g., buffer space, energy) to carry the content until delivery. The carrier node uses the above mentioned criteria to calculate a utility value. Afterwards, it considers itself and all of its one-hop neighbours as the potential forwarder and selects the node with the highest utility value as the forwarder. The selected forwarder node then carries the content, and if it meets another node with higher utility value then forwards the content to that node. Thus the selection process can be formulated as a maximization problem as,

$$
\begin{aligned}
\text { select } & k \\
\text { maximizing } & U_{k, d}^{\alpha} \\
\text { s.t. } & E^{k}>E_{m i n}^{\alpha} \quad \text { and } B^{k}>B_{m i n}^{\alpha}
\end{aligned}
$$

Here, $U_{k, d}^{\alpha}$ represents node $k$ 's utility to forward content $\alpha$ to destination node (i.e., content requester) $d . E^{k}$ and $E_{m i n}^{\alpha}$ represent energy available to node $k$ and minimum energy required to forward $\alpha$, respectively. $B^{k}$ and $B_{\min }^{\alpha}$ represent the buffer space available to node $k$ and the minimum space required to store $\alpha$.

A node's ability to successfully deliver a content depends on its expected co-location probability with the destination node. Here, co-location refers to staying in the same POI, but not necessarily within each other's communication range. If both the forwarder and the destination nodes are co-located for longer time, the probability of contact opportunity and successful delivery increases. Moreover, the forwarder node needs sufficient neighbours to ensure it can deliver the content which is calculated using its connectivity value. Considering all these factors the utility value is calculated as,

$$
U_{k, d}^{\alpha}= \begin{cases}1, & \text { if } h_{k, d}=1 \\ L_{k, d}^{\alpha} C^{k}, & \text { otherwise }\end{cases}
$$


Here, $h_{k, d}$ shows the hop-distance between node $k$ and $d$. $L_{k, d}$ represents the co-location stay probability of the forwarder $(k)$ and the destination node $(d)$, and $C^{k}$ is the connectivity value of node $k$. The co-location stay probability is calculated as,

$$
L_{k, d}=\beta L_{k, d}^{c}+(1-\beta) L_{k, d}^{n}
$$

In Eq.(3), $L_{k, d}^{c}$ shows the co-location stay probability of node $k$ and $d$ in the current POI, and $L_{k, d}^{n}$ depicts the same in the next POI. $L_{k, d}^{n}$ is considered as the requester might soon finish its current activity and moves into the next POI, before obtaining the content. In that case, the content can still be delivered by a forwarder moving to the same POI, if it meets the requester there before the lifetime of the request expires. $\beta$ is used as a weighting factor. The co-location stay probability of node $k$ and node $d$ at current POI $c$ is determined as,

$$
L_{k, d}^{c}= \begin{cases}1, & \text { if } T_{k}^{c} \geq T_{d}^{c} \\ \frac{T_{k}^{c}}{T_{d}^{c}}, & \text { otherwise }\end{cases}
$$

Here, $T_{k}^{c}$ and $T_{d}^{c}$ shows the remaining stay time of node $k$ and $d$ in the current POI, respectively. Remaining stay time of a node is calculated using the probability density function (pdf) of stay time of nodes in a POI, and time already spent in that POI. Historical information can be used to create a personalized pdf if sufficient information is available. For example, if a user has visited a particular or similar tourist spots many times, and stayed in different POIs, duration of those stay times can be stored by the content sharing app to calculate such pdfs, which reflects personal trend and is more accurate. Xia et al. [16] has reported that stay time in different POIs follow different distributions which are mostly Log-normal, Gamma or Weibull. Without loss of generality we consider that stay time follows a Log-normal distribution, however, any proper pdf can be used in our formulation. From the distribution, the expected stay time of user $k$ at POI $c$ can be calculated as,

$$
\delta_{k}^{c}=e^{\mu+\frac{1}{2} \sigma^{2}}
$$

In the above equation $\mu$ and $\sigma$ represent the mean and standard deviation of stay time at POI $c$ which can be obtained if sufficient information about a tourist's stay time in that POI is available. Otherwise, a generalized average stay time can be obtained from statistics provided by the tourism research department [17]. Finally, remaining stay time at a POI $c$ can be calculated as,

$$
T_{k}^{c}=\delta_{k}^{c}-\gamma_{k}^{c}
$$

Here, $\gamma_{k}^{c}$ shows the time already spent by node $k$ in c. The value of $T_{d}^{c}$ can be automatically calculated by the content sharing app installed in a requester's device and added with the content request which is used to calculate the value of $L_{k, d}^{c}$ using Eq.(4). The value of $L_{k, d}^{n}$ can be calculated similarly using Eq. (4)-(6).
The weighting factor $\beta$ dictates which co-location stay probability (i.e., in the current POI or the next POI) should be given more priority and calculated as,

$$
\beta= \begin{cases}1, & \text { if } T_{d}^{c} \geq \Psi_{\alpha} \\ \frac{T_{d}^{c}}{\Psi_{\alpha}}, & \text { otherwise }\end{cases}
$$

Here, $\Psi_{\alpha}$ represents the expiry time of the content request $\alpha$. If the destination node stays longer in the current POI than the request's life time then co-location stay probability in the current POI should be given the highest weight, otherwise nodes $k$ and $d$ have the possibility to meet in the next POI and the weight is determined by the ratio of remaining stay time and request lifetime.

The connectivity value measures the degree of association of a node with its immediate and multi-hop neighbours and is calculated using a neighbourhood table as in [9]. Each node periodically broadcasts its neighbour list with the hello message which is used by other nodes to update their neighbourhood table. From the neighbourhood table, node $k$ considers the number of $n$-hop neighbours seen within the last $t$ time units and calculates its connectivity value as,

$$
C^{k}=\log \left(1+h_{1}+\frac{h_{2}}{2}+\frac{h_{3}}{3} \ldots+\frac{h_{n}}{n}\right) / \log (M)
$$

Here, $h^{n}$ represents the number of $n$-hop neighbours of node $k . M$ is considered as the maximum number of people seen in a tourist spot and the logarithm in the denominator is used for normalization purpose. The value of $M$ can be obtained using the data from tourism department [17]. A lower value of $n$ misses relevant information and a higher value introduces more traffic as the size of the neighbourhood table to be broadcast increases. As a trade-off 2-hop connectivity $(n=2)$ is used as in [18]. The logarithm function in the numerator ensure diminishing marginal utility widely used in microeconomics. Node $k$ calculates its available energy as,

$$
E^{k}=E_{r}^{k} / E_{f}^{k}
$$

Here, $E_{r}^{k}$ shows $k$ 's remaining energy and $E_{f}^{k}$ is its full energy. The minimum energy required to forward/deliver content $\alpha$ can be calculated as,

$$
E_{\text {min }}^{\alpha}=\left(E_{u}^{k}+E^{\alpha}\right) / E_{f}^{\kappa}
$$

Here, $E_{u}^{k}$ represents the energy consumption due to regular use (e.g., call, sms, apps) and $E^{\alpha}$ shows the energy required to deliver content $\alpha$. The content sharing app can periodically update the energy usage statistics to calculate energy drain $E_{t}^{k}$ in each time unit due to regular usage. Similar to the work proposed in [19], we consider inter-contact time among nodes are exponentially distributed with parameter $\lambda$. Therefore, we can multiply $E_{t}^{k}$ with $1 / \lambda$, which is the time the node $k$ is expected to wait before it meets another forwarder or destination node, to obtain energy drain due to regular use $\left(E_{u}^{k}\right)$. The content sharing app can estimate $\lambda$ from past time window. The energy required to deliver content $\alpha$ can be 
obtained by multiplying the size of $\alpha$ with energy required to transfer single unit of data. Node $k$ can calculate this required energy from past experience of data transfer.

Users specify the amount of storage they are willing to allocate for the content sharing app and also for carrying other users' request. By default any suitable value can be used by the content sharing app. Node $k$ checks the available storage space and the size of content $\alpha$ to decide whether to carry the content or not.

Finally, node $k$ calculates its utility value using Eq. (2) and report it back to the content carrier node. Upon receiving utility value from all of its one-hop neighbour, the carrier node checks who has the highest utility using Eq. (1). If the carrier node itself has the highest utility it continues to carry the content, otherwise it forwards the content to the node offering highest utility. In case of a tie, the carrier node first checks the available energy and then the buffer space to take forwarding decision. If all the values are same then ties are broken by selecting a random node. This way successive carrier nodes carry the content until it is successfully delivered or the request lifetime expires. Algorithm 1 outlines the message forwarding process which is initiated when a carrier node $A$ receives content or meets a new node.

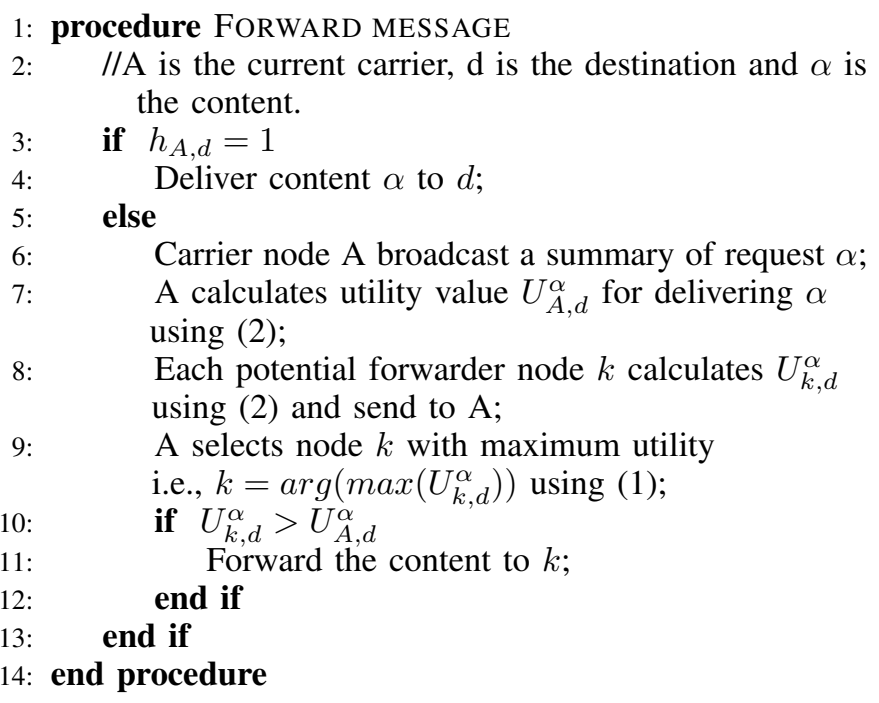

Algorithm 1: Message forwarding Procedure

\section{PERFormance EVAluation}

\section{A. Simulation setup}

To the best of our knowledge, there is no publicly available dataset demonstrating movement of tourist in a tourist spot. Therefore, we generated synthetic mobility data as in [8], [9]. We selected Lakes Entrance, one of the most popular tourist spots in Victoria, Australia as the test location. Different POIs inside the tourist spot \{'Accommodation', 'Bowling', 'Camping', 'Fishing', 'Food and Shopping', 'Walking' $\}$ were marked using information from tourist portals. In simulation, tourists entered the area using the Poisson distribution and stayed there using the negative binomial distribution which is consistent with the characteristics of tourists [20]. Movement inside a POI was dictated by the nature of the activity available in that POI. For example, inside the 'Fishing' region a node randomly selected a particular point and stayed at that point for a random amount of time before moving to some other point. Such characteristics are consistent with fishing activities in real life settings where people start catching fish at a particular location, and then either stay at that location for the whole duration or change locations if fishes are not available. These properties were considered while generating the synthetic mobility data. Nodes generated content request based on their interest using Zipf distribution as in [19]. NS3 was used as the simulation platform. Some simulation parameters are listed in Table I.

TABLE I: Simulation parameter

\begin{tabular}{|c|c|}
\hline Simulation parameters & Value \\
\hline Simulation area & $10 \mathrm{Km} \mathrm{X} \mathrm{5} \mathrm{Km}$ \\
\hline Number of nodes & 150 \\
\hline Duration & 3 days \\
\hline Avg. node arrival & $5-25 \mathrm{node} / \mathrm{hr}$ \\
\hline Range of IEEE $802.11 \mathrm{n}$ communication & $150 \mathrm{~m}$ \\
\hline Content size & $10 \mathrm{~KB}-2 \mathrm{MB}$ \\
\hline
\end{tabular}

\section{B. Simulation results}

To measure the performance of the proposed UBF approach three metrics were used, namely (i) hit rate, (ii) delivery success rate and (iii) average delivery latency. Hit rate and delivery success rate show the percentage of the requests for which contents are successfully located and received by the requesters, respectively. The average delivery latency is the average time required to obtain contents. The proposed UBF approach is compared with the approach proposed in [9] denoted as 'CEMT' throughout this section. We also compared UBF with SPOON approach proposed in [6] which uses interest similarity and meeting frequency of users for group creation and degree centrality measurement for coordinator (i.e., administrator) selection. It also uses interest oriented routing for message forwarding. As SPOON [6] was originally proposed for content sharing in work-place type scenarios, we applied the characteristics of a tourist spot into SPOON for obtaining results. A variation of SPOON [6] is used for comparison too, which uses similar group formation and administrator selection as in SPOON [6], but uses spray-andwait routing protocol for message forwarding. This approach is denoted as 'SPOON-SW' in the rest of the paper.

The effect of different node arrival rate is presented in Fig. $2 \mathrm{a}$ and $2 \mathrm{~b}$. Both figures show that the hit and success rates increase with a higher node arrival rate as more nodes are available to participate in the sharing process. Proposed UBF approach achieves the highest rate in all the cases with a hit rate of $79.82 \%$ and success rate of $77.50 \%$ compared to $75.39 \%$ hit rate and $69.17 \%$ success rate achieved by CEMT [9] approach for an arrival rate of 25 nodes/hr. In comparison, SPOON and SPOON-SW achieves much lower hit $(61.94 \%$ and $57.21 \%$, respectively) and success rate $(60.27 \%$ and $57.07 \%$, respectively) for the same arrival rate (i.e., 25 nodes/hr). The UBF approach achieves higher hit and success rate due to the proposed forwarder node selection policy based on co-location stay time, connectivity value and available resources. A higher co-location stay time increases the probability of having the selected forwarder and the destination 


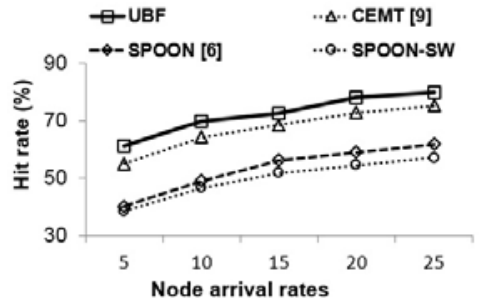

(a)

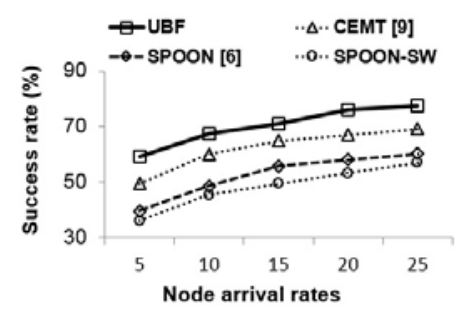

(b)

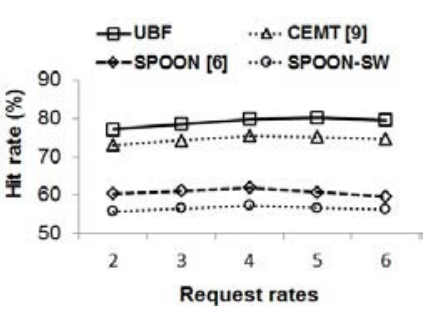

(c)

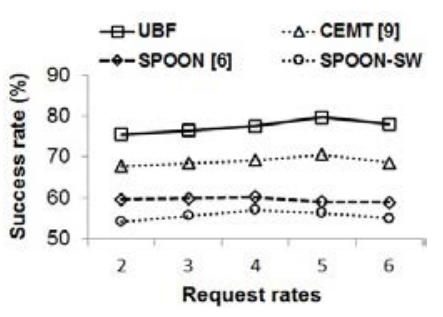

(d)

Fig. 2: (a) Hit rate and (b) success rate for different node arrival rates (number of arriving nodes/hr) keeping the average per node content request rate fixed at 4 requests/hr; (c) hit rate and (d) success rate for different request rates (number of requests/hr) keeping node arrival rate fixed at 25 nodes/hr

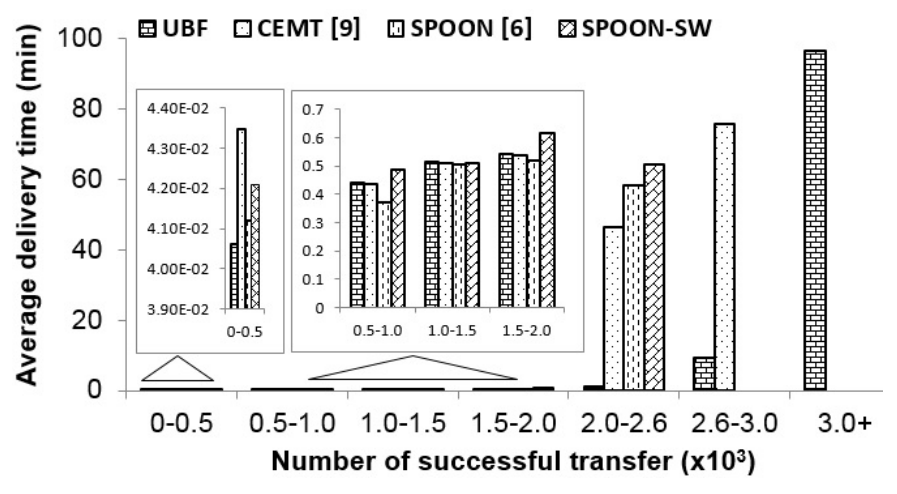

Fig. 3: Average delivery latency

node staying in the same POI for longer time, and hence more opportunistic contact; a higher connectivity value ensures that the selected forwarder node will have more neighbours to facilitate successful delivery, and available resource ensures the forwarder is capable of carrying the content for longer period of time.

Fig. $2 c$ and $2 d$ represent the impact of varying request rates. It shows that hit and success rates start to increase with an increasing request rate. This is because, at a higher request rate, nodes from the same neighbourhood are likely to generate more requests for the same content which, in turn, enable them to successfully get that content from a neighbour who has already requested and received that content. However, after a certain request rate $(5 \mathrm{req} / \mathrm{hr})$ both rates start to decrease as the network becomes congested with a higher request rate. UBF approach achieves $79.58 \%$ hit rate and $78.02 \%$ success rate even when the request rate is very high $(6 \mathrm{req} / \mathrm{hr})$. In contrast, other approaches achieves comparatively lower hit (56-74\%) and success rate (55-68\%) for the same request rate (6 req/hr). Existing literature proposed for work-place type scenarios reported hit and success rates within the range of $75-90 \%$ [3], [6]. In that regard, our method achieves good performance in tourist destinations where movement patterns are irregular and social ties are mostly non-existent.

Fig. 3 represents the average latency for successfully receiving contents. The latency values for successful deliveries are sorted in ascending order and averaged over each 500 deliveries for convenience of presentation. All the approaches can complete up to $2 \times 10^{3}$ successful transfers within a short time (e.g., up to $0.54 \mathrm{mins}$ (UBF), $0.53 \mathrm{mins}$ (CEMT), 0.52 mins (SPOON) and $0.61 \mathrm{mins}(\mathrm{SPOON}-\mathrm{SW})$ ). Afterwards from $2 \times 10^{3}$ to $2.6 \times 10^{3}$ requests, the UBF approach shows significantly lower delays (e.g., UBF 1.18 mins, CEMT 46.43 mins, SPOON 58.13 mins and SPOON-SW 64.07 mins) compared to other approaches. The reason for getting a comparatively lower delay for UBF approach is that it selects a forwarder node with higher connectivity, and hence has a better opportunity of delivering the content faster through more neighbours. The figure also suggests that the UBF approach is capable of managing a high number of requests and delivering contents even if the delay is longer for some of the requests where other approaches fail altogether. Such long delays are acceptable for a small number of requests considering the characteristics of the environments under investigation (i.e., tourist spots) where people usually stay for few hours to couple of days, and delivery is more important than delay.

The impact of various mobility patterns inside different POIs on hit and success rates for the proposed UBF approach are shown in Fig. 4, The figure shows that 'Bowling' achieves the highest hit and success rate among all. In comparison, 'Camping', 'Fishing' and 'Walking' attains relatively lower hit and success rates. 'Bowling' was considered as an indoor activity constrained in a smaller area, hence users were within the communication range of each other more often and successfully shared contents among themselves. On the other

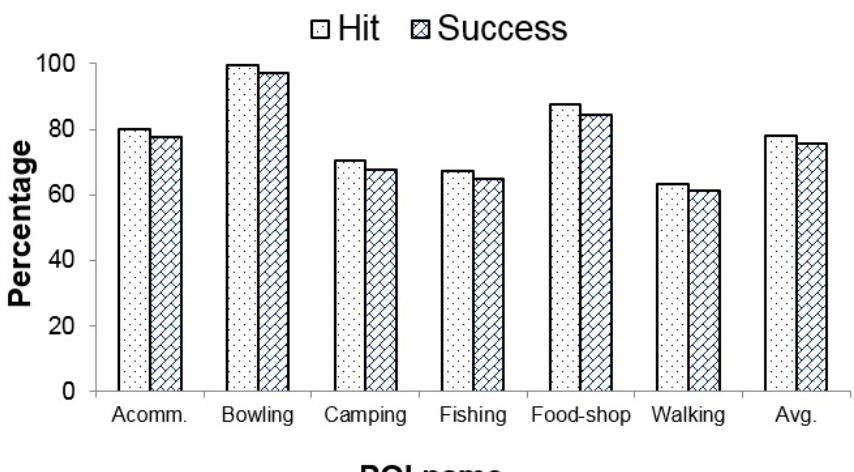

Fig. 4: Hit rate and Success rate in different POIs 


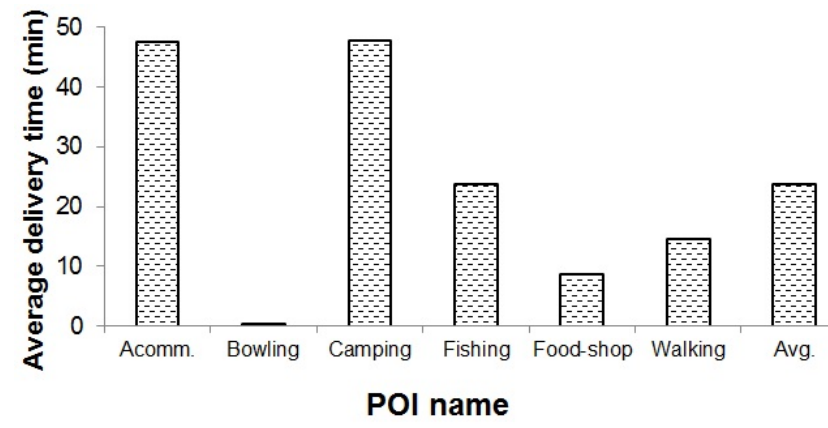

Fig. 5: Average delivery latency in different POIs

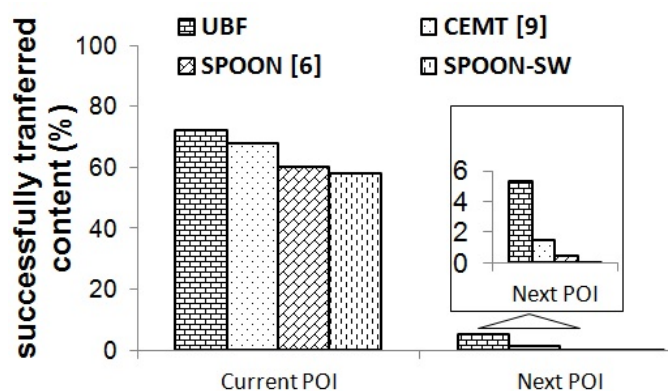

Fig. 6: Percentage of successfully delivered requests and colocation of nodes

hand, a larger area and low mobility resulted in lower hit and success rates for 'Camping' and 'Fishing'. In comparison, 'Walking' was a more dynamic activity and due to high mobility of the nodes fixed along a trail produced low hit and success rates.

The average delivery latency in different POIs is presented in Fig. 5. It suggests that 'Bowling' is accounted for the lowest latency since it is considered as an activity inside a smaller area as discussed before. On the other hand, 'Accommodation' and 'Camping' suffers from higher delay due to having a large area and low mobility that leads to infrequent contacts, and hence longer delay. Note that for $92.5 \%$ of the successful deliveries, the latency is very low as shown in the first part of Fig. 3 (up to $3 \times 10^{3}$ requests). The objective of this figure is to show the variation of latency across various POIs.

The proposed UBF approach uses co-location stay probability in the current POI as well as in next POI for selecting the forwarder node. The impact of such selection policy is presented in Fig. 6. It shows that the UBF approach achieves higher successful deliveries in the current POI (UBF $72.16 \%$ vs. others $67.74 \% \max$ ) as well as in the next POI (UBF 5.34\% vs. others $1.43 \% \max$ ). The reason for obtaining a higher rate is that the node offering a higher co-location stay time with the destination is selected as the forwarder node which, in turn, ensures that the forwarder node will stay in the same POI as the destination node, even if one of them moves to the next POI before successful delivery.

\section{CONCLUSION}

Message forwarding plays a major role for successful content delivery in decentralized content sharing. In this article, we employed a utility based message forwarding scheme using co-location stay time, connectivity value, available energy and buffer space. Simulation results demonstrate that the proposed UBF approach achieves higher hit and success rate, and lower latency compared to other approaches. The UBF approach also alleviates the requirement of having a predictable movement pattern or pre-existing social relationship, which is primarily used in the existing literature and mostly non-existent in environment under consideration (i.e., tourist destinations).

\section{REFERENCES}

[1] http://www.cisco.com/c/en/us/solutions/collateral/serviceprovider/visual-networking-index-vni/mobile-white-paper-c11520862.html.

[2] http://arstechnica.com/business/2012/05/bandwidth-explosion-asinternet-use-soars-can-bottlenecks-be-averted/.

[3] E. Talipov, Y. Chon, and H. Cha, "Content sharing over smartphonebased delay-tolerant networks," IEEE Mobile Computing, vol. 12, no. 3, pp. 581-595, 2013.

[4] A. Vahdat and D. Becker, "Epidemic routing for partially connected ad hoc networks," Technical Report CS-200006, Duke University, 2000.

[5] Q. Yuan, I. Cardei, and J. Wu, "Predict and relay: An efficient routing in disruption-tolerant networks," in ACM MobiHoc 2009, pp. 95-104.

[6] K. Chen, S. Member, H. Shen, S. Member, and H. Zhang, "Leveraging social networks for $\mathrm{p} 2 \mathrm{p}$ content-based file sharing in disconnected manets," IEEE Mobile Computing, vol. 13, no. 2, pp. 235-249, 2014.

[7] P. Hui, J. Crowcroft, and E. Yoneki, "Bubble rap: Social-based forwarding in delay-tolerant networks," IEEE Mobile Computing, vol. 10, no. 11, pp. 1576-1589, 2011.

[8] S. Kaisar, J. Kamruzzaman, G. Karmakar, and I. Gondal, "Content sharing among visitors with irregular movement patterns in visiting hotspots," in IEEE NCA 2015, pp. 230-234.

[9] S. Kaisar, J. Kamruzzaman, G. Karmakar, and I. Gondal, "Content exchange among mobile tourists using users' interest and place-centric activities," in IEEE ICICS 2015, pp. 1-5.

[10] T. Spyropoulos, K. Psounis, and C. S. Raghavendra, "Spray and wait: an efficient routing scheme for intermittently connected mobile networks," in ACM SIGCOMM 2005, pp. 252-259.

[11] A. Lindgren, A. Doria, and O. Scheln, "Probabilistic routing in intermittently connected networks," SIGMOBILE, vol. 7, no. 3, pp. 19-20.

[12] J. Leguay, T. Friedman, and V. Conan, "Dtn routing in a mobility pattern space," in ACM WDTN 2005, pp. 276-283.

[13] B. Burns, O. Brock, and B. N. Levine, "Mora routing and capacity building in disruption-tolerant networks," Elsevier Ad hoc networks, vol. 6, no. 4, pp. 600-620, 2008.

[14] E. M. Daly and M. Haahr, "Social network analysis for routing in disconnected delay-tolerant manets," in MobiHoc 2007, pp. 32-40.

[15] E. M. Daly and M. Haahr, "Social network analysis for information flow in disconnected delay-tolerant manets," IEEE Mobile Computing, vol. 8, no. 5, pp. 606-621, 2009.

[16] J. C. Xia, P. Zeephongsekul, and D. Packer, "Spatial and temporal modelling of tourist movements using semi-markov processes," Tourism Management, vol. 32, no. 4, pp. 844-851, 2011.

[17] http://www.tra.gov.au/research/regional.html.

[18] M. Grossglauser and D. Tse, "Mobility increases the capacity of ad-hoc wireless networks," in IEEE INFOCOM 2001, vol. 3, pp. 1360-1369.

[19] W. Gao, G. Cao, A. Iyengar, and M. Srivatsa, "Supporting cooperative caching in disruption tolerant networks," in ICDCS 2011, pp. 151-161.

[20] M. Kruger and M. Saayman, "The determinants of visitor length of stay at the kruger national park," koedoe, vol. 56, no. 2, pp. 1-11, 2014. 